\title{
Conformable fractional approximation by max-product operators
}

\author{
George A. Anastassiou
}

\begin{abstract}
Here we study the approximation of functions by a big variety of Maxproduct operators under conformable fractional differentiability. These are positive sublinear operators. Our study is based on our general results about positive sublinear operators. We produce Jackson type inequalities under conformable fractional initial conditions. So our approach is quantitative by producing inequalities with their right hand sides involving the modulus of continuity of a high order conformable fractional derivative of the function under approximation.
\end{abstract}

Mathematics Subject Classification (2010): 26A33, 41A17, 41A25, 41A36.

Keywords: positive sublinear operators, Max-product operators, modulus of continuity, conformable fractional derivative.

\section{Introduction}

The main motivation here is the monograph by B. Bede, L. Coroianu and S. Gal [4], 2016.

Let $N \in \mathbb{N}$, the well-known Bernstein polynomials ([7]) are positive linear operators, defined by the formula

$$
B_{N}(f)(x)=\sum_{k=0}^{N}\left(\begin{array}{c}
N \\
k
\end{array}\right) x^{k}(1-x)^{N-k} f\left(\frac{k}{N}\right), \quad x \in[0,1], f \in C([0,1]) .
$$

T. Popoviciu in [8], 1935, proved for $f \in C([0,1])$ that

$$
\left|B_{N}(f)(x)-f(x)\right| \leq \frac{5}{4} \omega_{1}\left(f, \frac{1}{\sqrt{N}}\right), \quad \forall x \in[0,1]
$$

where

$$
\omega_{1}(f, \delta)=\sup _{\substack{x, y \in[0,1]: \\|x-y| \leq \delta}}|f(x)-f(y)|, \quad \delta>0
$$


is the first modulus of continuity.

G.G. Lorentz in [7], 1986, p. 21, proved for $f \in C^{1}([0,1])$ that

$$
\left|B_{N}(f)(x)-f(x)\right| \leq \frac{3}{4 \sqrt{N}} \omega_{1}\left(f^{\prime}, \frac{1}{\sqrt{N}}\right), \quad \forall x \in[0,1],
$$

In [4], p. 10, the authors introduced the basic Max-product Bernstein operators,

$$
B_{N}^{(M)}(f)(x)=\frac{\bigvee_{k=0}^{N} p_{N, k}(x) f\left(\frac{k}{N}\right)}{\bigvee_{k=0}^{N} p_{N, k}(x)}, \quad N \in \mathbb{N},
$$

where $\bigvee$ stands for maximum, and

$$
p_{N, k}(x)=\left(\begin{array}{c}
N \\
k
\end{array}\right) x^{k}(1-x)^{N-k}
$$

and $f:[0,1] \rightarrow \mathbb{R}_{+}=[0, \infty)$.

These are nonlinear and piecewise rational operators.

The authors in [4] studied similar such nonlinear operators such as: the Maxproduct Favard-Szász-Mirakjan operators and their truncated version, the Maxproduct Baskakov operators and their truncated version, also many other similar specific operators. The study in [4] is based on presented there general theory of sublinear operators. These Max-product operators tend to converge faster to the on hand function.

So we mention from [4], p. 30, that for $f:[0,1] \rightarrow \mathbb{R}_{+}$continuous, we have the estimate

$$
\left|B_{N}^{(M)}(f)(x)-f(x)\right| \leq 12 \omega_{1}\left(f, \frac{1}{\sqrt{N+1}}\right), \text { for all } N \in \mathbb{N}, x \in[0,1],
$$

Also from [4], p. 36, we mention that for $f:[0,1] \rightarrow \mathbb{R}_{+}$being concave function we get that

$$
\left|B_{N}^{(M)}(f)(x)-f(x)\right| \leq 2 \omega_{1}\left(f, \frac{1}{N}\right), \text { for all } x \in[0,1],
$$

a much faster convergence.

In this article we expand the study in [4] by considering conformable fractional smoothness of functions. So our inequalities are with respect to $\omega_{1}\left(D_{\alpha}^{n} f, \delta\right), \delta>0$, $n \in \mathbb{N}$, where $D_{\alpha}^{n} f$ is the $n$th order conformable $\alpha$-fractional derivative, $\alpha \in(0,1]$, see $[1],[6]$.

We present at first some background and general related theory of sublinear operators and then we apply it to specific as above Max-product operators.

\section{Background}

We make

Definition 2.1. Let $f:[0, \infty) \rightarrow \mathbb{R}$ and $\alpha \in(0,1]$. We say that $f$ is an $\alpha$-fractional continuous function, iff $\forall \varepsilon>0 \exists \delta>0$ : for any $x, y \in[0, \infty)$ such that $\left|x^{\alpha}-y^{\alpha}\right| \leq \delta$ we get that $|f(x)-f(y)| \leq \varepsilon$.

We give 
Theorem 2.2. Over $[a, b] \subseteq[0, \infty), \alpha \in[0,1]$, a $\alpha$-fractional continuous function is a uniformly continuous function and vice versa, a uniformly continuous function is an $\alpha$-fractional continuous function.

(Theorem 2.2 is not valid over $[0, \infty)$.)

Note. Let $x, y \in[a, b] \subseteq[0, \infty)$, and $g(x)=x^{\alpha}, 0<\alpha \leq 1$, then

$$
g^{\prime}(x)=\alpha x^{\alpha-1}=\frac{\alpha}{x^{1-\alpha}}, \text { for } x \in(0, \infty) .
$$

Since $a \leq x \leq b$, then $\frac{1}{x} \geq \frac{1}{b}>0$ and $\frac{\alpha}{x^{1-\alpha}} \geq \frac{\alpha}{b^{1-\alpha}}>0$.

Assume $y>x$. By the mean value theorem we get

$$
y^{\alpha}-x^{\alpha}=\frac{\alpha}{\xi^{1-\alpha}}(y-x), \text { where } \xi \in(x, y) .
$$

A similar to (2.1) equality when $x>y$ is true.

Then we obtain

$$
\frac{\alpha}{b^{1-\alpha}}|y-x| \leq\left|y^{\alpha}-x^{\alpha}\right|=\frac{\alpha}{\xi^{1-\alpha}}|y-x| .
$$

Thus, it holds

$$
\frac{\alpha}{b^{1-\alpha}}|y-x| \leq\left|y^{\alpha}-x^{\alpha}\right|
$$

Proof of Theorem 2.2.

$(\Rightarrow)$ Assume that $f$ is $\alpha$-fractional continuous function on $[a, b] \subseteq[0, \infty)$. It means $\forall \varepsilon>0 \exists \delta>0$ : whenever $x, y \in[a, b]:\left|x^{\alpha}-y^{\alpha}\right| \leq \delta$, then $|f(x)-f(y)| \leq \varepsilon$. Let for $\left\{x_{n}\right\}_{n \in \mathbb{N}} \in[a, b]:\left\{x_{n} \rightarrow \lambda \in[a, b] \Leftrightarrow x_{n}^{\alpha} \rightarrow \lambda^{\alpha}\right\}$, it implies $f\left(x_{n}\right) \rightarrow f(\lambda)$, therefore $f$ is continuous in $\lambda$. Therefore $f$ is uniformly continuous over $[a, b]$.

For the converse we use the following criterion:

Lemma 2.3. A necessary and sufficient condition that the function $f$ is not $\alpha$-fractional continuous $(\alpha \in(0,1])$ over $[a, b] \subseteq[0, \infty)$ is that there exist $\varepsilon_{0}>0$, and two sequences $X=\left(x_{n}\right), Y=\left(y_{n}\right)$ in $[a, b]$ such that if $n \in \mathbb{N}$, then $\left|x_{n}^{\alpha}-y_{n}^{\alpha}\right| \leq \frac{1}{n}$ and $\left|f\left(x_{n}\right)-f\left(y_{n}\right)\right|>\varepsilon_{0}$.

Proof. Obvious.

(Proof of Theorem 2.2 continuous) $(\Leftarrow)$ Uniform continuity implies $\alpha$-fractional continuity on $[a, b] \subseteq[0,+\infty)$. Indeed: let $f$ uniformly continuous on $[a, b]$, hence $f$ continuous on $[a, b]$. Assume that $f$ is not $\alpha$-fractional continuous on $[a, b]$. Then by Lemma 2.3 there exist $\varepsilon_{0}>0$, and two sequences $X=\left(x_{n}\right), Y=\left(y_{n}\right)$ in $[a, b]$ such that if $n \in \mathbb{N}$, then $\left|x_{n}^{\alpha}-y_{n}^{\alpha}\right| \leq \frac{1}{n}$ and

$$
\left|f\left(x_{n}\right)-f\left(y_{n}\right)\right|>\varepsilon_{0} .
$$

Since $[a, b]$ is compact, the sequences $\left\{x_{n}\right\},\left\{y_{n}\right\}$ are bounded. By the BolzanoWeierstrass theorem, there is a subsequence $\left\{x_{n(k)}\right\}$ of $\left\{x_{n}\right\}$ which converges to an element $z$. Since $[a, b]$ is closed, the limit $z \in[a, b]$, and $f$ is continuous at $z$.

We have also that

$$
\frac{\alpha}{b^{1-\alpha}}\left|x_{n}-y_{n}\right| \leq\left|x_{n}^{\alpha}-y_{n}^{\alpha}\right| \leq \frac{1}{n}
$$


hence

$$
\left|x_{n}-y_{n}\right| \leq \frac{b^{1-\alpha}}{\alpha n}
$$

It is clear that the corrsponding subsequence $\left(y_{n(k)}\right)$ of $Y$ also converges to $z$. Hence $f\left(x_{n(k)}\right) \rightarrow f(z)$, and $f\left(y_{n(k)}\right) \rightarrow f(z)$. Therefore, when $k$ is sufficiently large we have $\left|f\left(x_{n(k)}\right)-f\left(y_{n(k)}\right)\right|<\varepsilon_{0}$, contradicting (2.4).

We need

Definition 2.4. Let $[a, b] \subseteq[0, \infty), \alpha \in[0,1]$. We define the $\alpha$-fractional modulus of continuity:

$$
\omega_{1}^{\alpha}(f, \delta):=\sup _{\substack{x, y \in[a, b]: \\\left|x^{\alpha}-y^{\alpha}\right| \leq \delta}}|f(x)-f(y)|, \delta>0 .
$$

The same definition holds over $[0, \infty)$.

\section{Properties.}

1) $\omega_{1}^{\alpha}(f, 0)=0$.

2) $\omega_{1}^{\alpha}(f, \delta) \rightarrow 0$ as $\delta \downarrow 0$, iff $f$ is in the set of all $\alpha$-fractional continuous functions, denoted as $f \in C_{\alpha}([a, b], \mathbb{R})(=C([a, b], \mathbb{R}))$.

Proof. $(\Rightarrow)$ Let $\omega_{1}^{\alpha}(f, \delta) \rightarrow 0$ as $\delta \downarrow 0$. Then $\forall \varepsilon>0, \exists \delta>0$ with $\omega_{1}^{\alpha}(f, \delta) \leq \varepsilon$, i.e. $\forall x, y \in[a, b]:\left|x^{\alpha}-y^{\alpha}\right| \leq \delta$ we get $|f(x)-f(y)| \leq \varepsilon$. That is $f \in C_{\alpha}([a, b], \mathbb{R})$.

$(\Leftarrow)$ Let $f \in C_{\alpha}([a, b], \mathbb{R})$. Then $\forall \varepsilon>0, \exists \delta>0$ : whenever $\left|x^{\alpha}-y^{\alpha}\right| \leq \delta$, $x, y \in[a, b]$, it implies $|f(x)-f(y)| \leq \varepsilon$, i.e. $\forall \varepsilon>0, \exists \delta>0: \omega_{1}^{\alpha}(f, \delta) \leq \varepsilon$. That is $\omega_{1}^{\alpha}(f, \delta) \rightarrow 0$, as $\delta \downarrow 0$.

3) $\omega_{1}^{\alpha}$ is $\geq 0$ and non-decreasing on $\mathbb{R}_{+}$.

4) $\omega_{1}^{\alpha}$ is subadditive:

$$
\omega_{1}^{\alpha}\left(f, t_{1}+t_{2}\right) \leq \omega_{1}^{\alpha}\left(f, t_{1}\right)+\omega_{1}^{\alpha}\left(f, t_{2}\right) .
$$

Proof. If $\left|x^{\alpha}-y^{\alpha}\right| \leq t_{1}+t_{2}(x, y \in[a, b])$, there is a point $z \in[a, b]$ for which $\left|x^{\alpha}-z^{\alpha}\right| \leq t_{1},\left|y^{\alpha}-z^{\alpha}\right| \leq t_{2}$, and $|f(x)-f(y)| \leq|f(x)-f(z)|+|f(z)-f(y)| \leq$ $\omega_{1}^{\alpha}\left(f, t_{1}\right)+\omega_{1}^{\alpha}\left(f, t_{2}\right)$, implying $\omega_{1}^{\alpha}\left(f, t_{1}+t_{2}\right) \leq \omega_{1}^{\alpha}\left(f, t_{1}\right)+\omega_{1}^{\alpha}\left(f, t_{2}\right)$.

5) $\omega_{1}^{\alpha}$ is continuous on $\mathbb{R}_{+}$.

Proof. We get

$$
\left|\omega_{1}^{\alpha}\left(f, t_{1}+t_{2}\right)-\omega_{1}^{\alpha}\left(f, t_{1}\right)\right| \leq \omega_{1}^{\alpha}\left(f, t_{2}\right) .
$$

By properties 2), 3), 4), we get that $\omega_{1}^{\alpha}(f, t)$ is continuous at each $t \geq 0$.

6) Clearly it holds

$$
\omega_{1}^{\alpha}\left(f, t_{1}+\ldots+t_{n}\right) \leq \omega_{1}^{\alpha}\left(f, t_{1}\right)+\ldots+\omega_{1}^{\alpha}\left(f, t_{n}\right),
$$

for $t=t_{1}=\ldots=t_{n}$, we obtain

$$
\omega_{1}^{\alpha}(f, n t)=n \omega_{1}^{\alpha}(f, t) .
$$

7) Let $\lambda \geq 0, \lambda \notin \mathbb{N}$, we get

$$
\omega_{1}^{\alpha}(f, \lambda t) \leq(\lambda+1) \omega_{1}^{\alpha}(f, t) .
$$


Proof. Let $n \in \mathbb{Z}_{+}: n \leq \lambda<n+1$, we see that

$$
\omega_{1}^{\alpha}(f, \lambda t) \leq \omega_{1}^{\alpha}(f,(n+1) t) \leq(n+1) \omega_{1}^{\alpha}(f, t) \leq(\lambda+1) \omega_{1}^{\alpha}(f, t) .
$$

Properties 1), 3), 4), 6), 7) are valid also for $\omega_{1}^{\alpha}$ defined over $[0, \infty)$.

We notice that $\omega_{1}^{\alpha}(f, \delta)$ is finite when $f$ is uniformly continuous on $[a, b]$. If $f:[0, \infty) \rightarrow \mathbb{R}$ is bounded then $\omega_{1}^{\alpha}(f, \delta)$ is again finite.

We need

Definition 2.5. ([1], [6]) Let $f:[0, \infty) \rightarrow \mathbb{R}$. The conformable $\alpha$-fractional derivative for $\alpha \in(0,1]$ is given by

$$
\begin{gathered}
D_{\alpha} f(t):=\lim _{\varepsilon \rightarrow 0} \frac{f\left(t+\varepsilon t^{1-\alpha}\right)-f(t)}{\varepsilon}, \\
D_{\alpha} f(0)=\lim _{t \rightarrow 0+} D_{\alpha} f(t) .
\end{gathered}
$$

If $f$ is differentiable, then

$$
D_{\alpha} f(t)=t^{1-\alpha} f^{\prime}(t),
$$

where $f^{\prime}$ is the usual derivative.

We define $D_{\alpha}^{n} f=D_{\alpha}^{n-1}\left(D_{\alpha} f\right)$.

If $f:[0, \infty) \rightarrow \mathbb{R}$ is $\alpha$-differentiable at $t_{0}>0, \alpha \in(0,1]$, then $f$ is continuous at $t_{0}$, see $[6]$.

We will use

Theorem 2.6. (see [3]) (Taylor formula) Let $\alpha \in(0,1]$ and $n \in \mathbb{N}$. Suppose $f$ is $(n+1)$ times conformable $\alpha$-fractional differentiable on $[0, \infty)$, and $s, t \in[0, \infty)$, and $D_{\alpha}^{n+1} f$ is assumed to be continuous on $[0, \infty)$. Then we have

$$
f(t)=\sum_{k=0}^{n} \frac{1}{k !}\left(\frac{t^{\alpha}-s^{\alpha}}{\alpha}\right)^{k} D_{\alpha}^{k} f(s)+\frac{1}{n !} \int_{s}^{t}\left(\frac{t^{\alpha}-\tau^{\alpha}}{\alpha}\right)^{n} D_{\alpha}^{n+1} f(\tau) \tau^{a-1} d \tau .
$$

The case $n=0$ follows.

Corollary 2.7. Let $\alpha \in(0,1]$. Suppose $f$ is $\alpha$-fractional differentiable on $[0, \infty)$, and $s, t \in[0, \infty)$. Assume that $D_{\alpha} f$ is continuous on $[0, \infty)$. Then

$$
f(t)=f(s)+\int_{s}^{t} D_{\alpha} f(\tau) \tau^{a-1} d \tau .
$$

Note. Theorem 2.6 and Corollary 2.7 are also true for $f:[a, b] \rightarrow \mathbb{R},[a, b] \subseteq[0, \infty)$, $s, t \in[a, b]$.

Proof of Corollary 2.7. Denote $I_{\alpha}^{s}(f)(t):=\int_{s}^{t} x^{\alpha-1} f(x) d x$. By [6] we get that

$$
D_{\alpha} I_{\alpha}^{s}(f)(t)=f(t), \text { for } t \geq s,
$$

where $f$ is any continuous function in the domain of $I_{\alpha}, \alpha \in(0,1)$.

Assume that $D_{\alpha} f$ is continuous, then

$$
D_{\alpha} I_{\alpha}^{s}\left(D_{\alpha} f\right)(t)=\left(D_{\alpha} f\right)(t), \forall t \geq s .
$$

Then, by [5], there exists a constant $c$ such that

$$
I_{\alpha}^{s}\left(D_{\alpha} f\right)(t)=f(t)+c .
$$


Hence

then $c=-f(s)$.

$$
0=I_{\alpha}^{s}\left(D_{\alpha} f\right)(s)=f(s)+c,
$$

Therefore

$$
I_{\alpha}^{s}\left(D_{\alpha} f\right)(t)=f(t)-f(s)=\int_{s}^{t}\left(D_{\alpha} f\right)(\tau) \tau^{\alpha-1} d \tau .
$$

The same proof applies for any $s \geq t$.

\section{Main results}

\section{We give}

Theorem 3.1. Let $\alpha \in(0,1]$ and $n \in \mathbb{Z}_{+}$. Suppose $f$ is $(n+1)$ times conformable $\alpha$-fractional differentiable on $[0, \infty)$, and $s, t \in[0, \infty)$, and $D_{\alpha}^{n+1} f$ is assumed to be continuous on $[0, \infty)$ and bounded. Then

$$
\left|f(t)-\sum_{k=0}^{n+1} \frac{1}{k !}\left(\frac{t^{\alpha}-s^{\alpha}}{\alpha}\right)^{k} D_{\alpha}^{k} f(s)\right| \leq \frac{\omega_{1}^{\alpha}\left(D_{\alpha}^{n+1} f, \delta\right)}{\alpha^{n+1}(n+1) !}\left|t^{\alpha}-s^{\alpha}\right|^{n+1}\left[1+\frac{\left|t^{\alpha}-s^{\alpha}\right|}{(n+2) \delta}\right],
$$

$\forall s, t \in[0, \infty), \delta>0$.

Note. Theorem 3.1 is valid also for $f:[a, b] \rightarrow \mathbb{R},[a, b] \subseteq \mathbb{R}_{+}$, any $s, t \in[a, b]$.

Proof. We have that

$$
\frac{1}{n !} \int_{s}^{t}\left(\frac{t^{\alpha}-\tau^{\alpha}}{\alpha}\right)^{n} D_{\alpha}^{n+1} f(s) \tau^{\alpha-1} d \tau=\frac{D_{\alpha}^{n+1} f(s)}{n !} \int_{s}^{t}\left(\frac{t^{\alpha}-\tau^{\alpha}}{\alpha}\right)^{n} \tau^{\alpha-1} d \tau
$$

(by $\frac{d \tau^{\alpha}}{d \tau}=\alpha \tau^{\alpha-1} \Rightarrow d \tau^{\alpha}=\alpha \tau^{\alpha-1} d \tau \Rightarrow \frac{1}{\alpha} d \tau^{\alpha}=\tau^{\alpha-1} d \tau$ )

$$
=\frac{D_{\alpha}^{n+1} f(s)}{\alpha^{n+1} n !} \int_{s}^{t}\left(t^{\alpha}-\tau^{\alpha}\right)^{n} d \tau^{\alpha}
$$

(by $\left.t \leq \tau \leq s \Rightarrow t^{\alpha} \leq \tau^{\alpha}(=: z) \leq s^{\alpha}\right)$

$$
\begin{gathered}
=\frac{D_{\alpha}^{n+1} f(s)}{\alpha^{n+1} n !} \int_{s^{\alpha}}^{t^{\alpha}}\left(t^{\alpha}-z\right)^{n} d z=\frac{D_{\alpha}^{n+1} f(s)}{\alpha^{n+1} n !} \frac{\left(t^{\alpha}-s^{\alpha}\right)^{n+1}}{n+1} \\
=\frac{D_{\alpha}^{n+1} f(s)}{(n+1) !}\left(\frac{t^{\alpha}-s^{\alpha}}{\alpha}\right)^{n+1} .
\end{gathered}
$$

Therefore it holds

$$
\frac{1}{n !} \int_{s}^{t}\left(\frac{t^{\alpha}-\tau^{\alpha}}{\alpha}\right)^{n} D_{\alpha}^{n+1} f(s) \tau^{\alpha-1} d \tau=\frac{D_{\alpha}^{n+1} f(s)}{(n+1) !}\left(\frac{t^{\alpha}-s^{\alpha}}{\alpha}\right)^{n+1} .
$$

By (2.16) and (2.17) we get:

$$
f(t)=\sum_{k=0}^{n+1} \frac{1}{k !}\left(\frac{t^{\alpha}-s^{\alpha}}{\alpha}\right)^{k} D_{\alpha}^{k} f(s)+
$$




$$
\frac{1}{n !} \int_{s}^{t}\left(\frac{t^{\alpha}-\tau^{\alpha}}{\alpha}\right)^{n}\left(D_{\alpha}^{n+1} f(\tau)-D_{\alpha}^{n+1} f(s)\right) \tau^{\alpha-1} d \tau
$$

Call the remainder as

$$
R_{n}(s, t):=\frac{1}{n !} \int_{s}^{t}\left(\frac{t^{\alpha}-\tau^{\alpha}}{\alpha}\right)^{n}\left(D_{\alpha}^{n+1} f(\tau)-D_{\alpha}^{n+1} f(s)\right) \tau^{\alpha-1} d \tau
$$

We estimate $R_{n}(s, t)$.

Cases:

1) Let $t \geq s$. Then

$$
\begin{gathered}
\left|R_{n}(s, t)\right| \leq \frac{1}{n !} \int_{s}^{t}\left(\frac{t^{\alpha}-\tau^{\alpha}}{\alpha}\right)^{n}\left|D_{\alpha}^{n+1} f(\tau)-D_{\alpha}^{n+1} f(s)\right| \tau^{\alpha-1} d \tau \\
\leq \frac{1}{\alpha n !} \int_{s}^{t}\left(\frac{t^{\alpha}-\tau^{\alpha}}{\alpha}\right)^{n} \omega_{1}^{\alpha}\left(D_{\alpha}^{n+1} f, \tau^{\alpha}-s^{\alpha}\right) d \tau^{\alpha} \\
=\frac{1}{\alpha^{n+1} n !} \int_{s}^{t}\left(t^{\alpha}-\tau^{\alpha}\right)^{n} \omega_{1}^{\alpha}\left(D_{\alpha}^{n+1} f, \frac{\delta\left(\tau^{\alpha}-s^{\alpha}\right)}{\delta}\right) d \tau^{\alpha}
\end{gathered}
$$

$(\delta>0)$

$$
\begin{gathered}
\leq \frac{\omega_{1}^{\alpha}\left(D_{\alpha}^{n+1} f, \delta\right)}{\alpha^{n+1} n !} \int_{s}^{t}\left(t^{\alpha}-\tau^{\alpha}\right)^{n}\left(1+\frac{\tau^{\alpha}-s^{\alpha}}{\delta}\right) d \tau^{\alpha} \\
=\frac{\omega_{1}^{\alpha}\left(D_{\alpha}^{n+1} f, \delta\right)}{\alpha^{n+1} n !} \int_{s^{\alpha}}^{t^{\alpha}}\left(t^{\alpha}-z\right)^{n}\left(1+\frac{z-s^{\alpha}}{\delta}\right) d z \\
=\frac{\omega_{1}^{\alpha}\left(D_{\alpha}^{n+1} f, \delta\right)}{\alpha^{n+1} n !}\left[\int_{s^{\alpha}}^{t^{\alpha}}\left(t^{\alpha}-z\right)^{n} d z+\frac{1}{\delta} \int_{s^{\alpha}}^{t^{\alpha}}\left(t^{\alpha}-z\right)^{(n+1)-1}\left(z-s^{\alpha}\right)^{2-1} d z\right] \\
=\frac{\omega_{1}^{\alpha}\left(D_{\alpha}^{n+1} f, \delta\right)}{\alpha^{n+1} n !}\left[\frac{\left(t^{\alpha}-s^{\alpha}\right)^{n+1}}{n+1}+\frac{1}{\delta} \frac{\Gamma(n+1) \Gamma(2)}{\Gamma(n+3)}\left(t^{\alpha}-s^{\alpha}\right)^{n+2}\right] \\
=\frac{\omega_{1}^{\alpha}\left(D_{\alpha}^{n+1} f, \delta\right)}{\alpha^{n+1} n !}\left[\frac{\left(t^{\alpha}-s^{\alpha}\right)^{n+1}}{n+1}+\frac{1}{\delta} \frac{n !}{(n+2) !}\left(t^{\alpha}-s^{\alpha}\right)^{n+2}\right] \\
=\frac{\omega_{1}^{\alpha}\left(D_{\alpha}^{n+1} f, \delta\right)}{\alpha^{n+1} n !}\left[\frac{\left(t^{\alpha}-s^{\alpha}\right)^{n+1}}{n+1}+\frac{1}{\delta} \frac{\left(t^{\alpha}-s^{\alpha}\right)^{n+2}}{(n+1)(n+2)}\right] \\
=\frac{\omega_{1}^{\alpha}\left(D_{\alpha}^{n+1} f, \delta\right)}{\alpha^{n+1}(n+1) !}\left(t^{\alpha}-s^{\alpha}\right)^{n+1}\left[1+\frac{\left(t^{\alpha}-s^{\alpha}\right)}{(n+2) \delta}\right] .
\end{gathered}
$$

We have proved that (case of $t \geq s$ )

$$
\left|R_{n}(s, t)\right| \leq \frac{\omega_{1}^{\alpha}\left(D_{\alpha}^{n+1} f, \delta\right)}{\alpha^{n+1}(n+1) !}\left(t^{\alpha}-s^{\alpha}\right)^{n+1}\left[1+\frac{\left(t^{\alpha}-s^{\alpha}\right)}{(n+2) \delta}\right],
$$

where $\delta>0$.

2) case of $t \leq s$ : We have

$$
\left|R_{n}(s, t)\right| \leq \frac{1}{n !}\left|\int_{t}^{s}\left(\frac{t^{\alpha}-\tau^{\alpha}}{\alpha}\right)^{n}\left(D_{\alpha}^{n+1} f(\tau)-D_{\alpha}^{n+1} f(s)\right) \tau^{\alpha-1} d \tau\right|
$$




$$
\begin{aligned}
& \frac{1}{n !}\left|\int_{t}^{s}\left(\frac{\tau^{\alpha}-t^{\alpha}}{\alpha}\right)^{n}\left(D_{\alpha}^{n+1} f(\tau)-D_{\alpha}^{n+1} f(s)\right) \tau^{\alpha-1} d \tau\right| \\
& \leq \frac{1}{\alpha n !} \int_{t}^{s}\left(\frac{\tau^{\alpha}-t^{\alpha}}{\alpha}\right)^{n}\left|D_{\alpha}^{n+1} f(\tau)-D_{\alpha}^{n+1} f(s)\right| d \tau^{\alpha} \\
& =\frac{1}{\alpha^{n+1} n !} \int_{t}^{s}\left(\tau^{\alpha}-t^{\alpha}\right)^{n} \omega_{1}^{\alpha}\left(D_{\alpha}^{n+1} f, s^{\alpha}-\tau^{\alpha}\right) d \tau^{\alpha} \\
& \leq \frac{\omega_{1}^{\alpha}\left(D_{\alpha}^{n+1} f, \delta\right)}{\alpha^{n+1} n !} \int_{t}^{s}\left(\tau^{\alpha}-t^{\alpha}\right)^{n}\left(1+\frac{s^{\alpha}-\tau^{\alpha}}{\delta}\right) d \tau^{\alpha} \\
& =\frac{\omega_{1}^{\alpha}\left(D_{\alpha}^{n+1} f, \delta\right)}{\alpha^{n+1} n !} \int_{t^{\alpha}}^{s^{\alpha}}\left(z-t^{\alpha}\right)^{n}\left(1+\frac{s^{\alpha}-z}{\delta}\right) d z \\
& =\frac{\omega_{1}^{\alpha}\left(D_{\alpha}^{n+1} f, \delta\right)}{\alpha^{n+1} n !}\left[\int_{t^{\alpha}}^{s^{\alpha}}\left(z-t^{\alpha}\right)^{n} d z+\frac{1}{\delta} \int_{t^{\alpha}}^{s^{\alpha}}\left(s^{\alpha}-z\right)^{2-1}\left(z-t^{\alpha}\right)^{(n+1)-1} d z\right] \\
& =\frac{\omega_{1}^{\alpha}\left(D_{\alpha}^{n+1} f, \delta\right)}{\alpha^{n+1} n !}\left[\frac{\left(s^{\alpha}-t^{\alpha}\right)^{n+1}}{n+1}+\frac{1}{\delta} \frac{\Gamma(2) \Gamma(n+1)}{\Gamma(n+3)}\left(s^{\alpha}-t^{\alpha}\right)^{n+2}\right] \\
& =\frac{\omega_{1}^{\alpha}\left(D_{\alpha}^{n+1} f, \delta\right)}{\alpha^{n+1} n !}\left[\frac{\left(s^{\alpha}-t^{\alpha}\right)^{n+1}}{n+1}+\frac{1}{\delta} \frac{n !}{(n+2) !}\left(s^{\alpha}-t^{\alpha}\right)^{n+2}\right] \\
& =\frac{\omega_{1}^{\alpha}\left(D_{\alpha}^{n+1} f, \delta\right)}{\alpha^{n+1} n !}\left[\frac{\left(s^{\alpha}-t^{\alpha}\right)^{n+1}}{n+1}+\frac{1}{\delta} \frac{\left(s^{\alpha}-t^{\alpha}\right)^{n+2}}{(n+1)(n+2)}\right] \\
& =\frac{\omega_{1}^{\alpha}\left(D_{\alpha}^{n+1} f, \delta\right)}{\alpha^{n+1}(n+1) !}\left(s^{\alpha}-t^{\alpha}\right)^{n+1}\left[1+\frac{\left(s^{\alpha}-t^{\alpha}\right)}{(n+2) \delta}\right] .
\end{aligned}
$$

We have proved that $(t \leq s)$

$$
\left|R_{n}(s, t)\right| \leq \frac{\omega_{1}^{\alpha}\left(D_{\alpha}^{n+1} f, \delta\right)}{\alpha^{n+1}(n+1) !}\left(s^{\alpha}-t^{\alpha}\right)^{n+1}\left[1+\frac{\left(s^{\alpha}-t^{\alpha}\right)}{(n+2) \delta}\right]
$$

$\delta>0$.

Conclusion. We have proved that $(\delta>0)$

$$
\left|R_{n}(s, t)\right| \leq \frac{\omega_{1}^{\alpha}\left(D_{\alpha}^{n+1} f, \delta\right)}{\alpha^{n+1}(n+1) !}\left|t^{\alpha}-s^{\alpha}\right|^{n+1}\left[1+\frac{\left|t^{\alpha}-s^{\alpha}\right|}{(n+2) \delta}\right], \quad \forall s, t \in[0, \infty) .
$$

The proof of the theorem now is complete.

We proved that

Theorem 3.2. Let $\alpha \in(0,1], n \in \mathbb{N}$. Suppose $f$ is $n$ times conformable $\alpha$-fractional differentiable on $[a, b] \subseteq[0, \infty)$, and let any $s, t \in[a, b]$. Assume that $D_{\alpha}^{n} f$ is continuous on $[a, b]$. Then

$$
\left|f(t)-\sum_{k=0}^{n} \frac{1}{k !}\left(\frac{t^{\alpha}-s^{\alpha}}{\alpha}\right)^{k} D_{\alpha}^{k} f(s)\right| \leq \frac{\omega_{1}^{\alpha}\left(D_{\alpha}^{n} f, \delta\right)}{\alpha^{n} n !}\left|t^{\alpha}-s^{\alpha}\right|^{n}\left[1+\frac{\left|t^{\alpha}-s^{\alpha}\right|}{(n+1) \delta}\right],
$$

where $\delta>0$. 
Proof. By Theorem 3.1.

Corollary 3.3. ( $n=1$ case of Theorem 3.2) Let $\alpha \in(0,1]$. Suppose $f$ is $\alpha$-conformable fractional differentiable on $[a, b] \subseteq[0, \infty)$, and let any $s, t \in[a, b]$. Assume that $D_{\alpha} f$ is continuous on $[a, b]$. Then

$$
\left|f(t)-f(s)-\left(\frac{t^{\alpha}-s^{\alpha}}{\alpha}\right) D_{\alpha} f(s)\right| \leq \frac{\omega_{1}^{\alpha}\left(D_{\alpha} f, \delta\right)}{\alpha}\left|t^{\alpha}-s^{\alpha}\right|\left[1+\frac{\left|t^{\alpha}-s^{\alpha}\right|}{2 \delta}\right],
$$

where $\delta>0$.

Corollary 3.4. (to Theorem 3.2) Same assumptions as in Theorem 3.2. For specific $s \in[a, b]$ assume that $D_{\alpha}^{k} f(s)=0, k=1, \ldots, n$. Then

$$
|f(t)-f(s)| \leq \frac{\omega_{1}^{\alpha}\left(D_{\alpha}^{n} f, \delta\right)}{\alpha^{n} n !}\left|t^{\alpha}-s^{\alpha}\right|^{n}\left[1+\frac{\left|t^{\alpha}-s^{\alpha}\right|}{(n+1) \delta}\right], \quad \delta>0 .
$$

The case $n=1$ follows:

Corollary 3.5. (to Corollary 3.4) For specific $s \in[a, b]$ assume that $D_{\alpha} f(s)=0$. Then

$$
|f(t)-f(s)| \leq \frac{\omega_{1}^{\alpha}\left(D_{\alpha} f, \delta\right)}{\alpha}\left|t^{\alpha}-s^{\alpha}\right|\left[1+\frac{\left|t^{\alpha}-s^{\alpha}\right|}{2 \delta}\right], \quad \delta>0 .
$$

We make

Remark 3.6. For $0<\alpha \leq 1, t, s \geq 0$, we have

$$
2^{\alpha-1}\left(x^{\alpha}+y^{\alpha}\right) \leq(x+y)^{\alpha} \leq x^{\alpha}+y^{\alpha} .
$$

Assume that $t>s$, then

$$
t=t-s+s \Rightarrow t^{\alpha}=(t-s+s)^{\alpha} \leq(t-s)^{\alpha}+s^{\alpha},
$$

hence $t^{\alpha}-s^{\alpha} \leq(t-s)^{\alpha}$.

Similarly, when $s>t \Rightarrow s^{\alpha}-t^{\alpha} \leq(s-t)^{\alpha}$.

Therefore it holds

$$
\left|t^{\alpha}-s^{\alpha}\right| \leq|t-s|^{\alpha}, \forall t, s \in[0, \infty) .
$$

Corollary 3.7. (to Theorem 3.2) Same assumptions as in Theorem 3.2. For specific $s \in[a, b]$ assume that $D_{\alpha}^{k} f(s)=0, k=1, \ldots, n$. Then

$$
|f(t)-f(s)| \leq \frac{\omega_{1}^{\alpha}\left(D_{\alpha}^{n} f, \delta\right)}{\alpha^{n} n !}|t-s|^{n \alpha}\left[1+\frac{|t-s|^{\alpha}}{(n+1) \delta}\right], \quad \delta>0,
$$

$\forall t \in[a, b] \subseteq[0, \infty)$

Corollary 3.8. (to Corollary 3.3) Same assumptions as in Corollary 3.3. For specific $s \in[a, b]$ assume that $D_{\alpha} f(s)=0$. Then

$$
|f(t)-f(s)| \leq \frac{\omega_{1}^{\alpha}\left(D_{\alpha} f, \delta\right)}{\alpha}|t-s|^{\alpha}\left[1+\frac{|t-s|^{\alpha}}{2 \delta}\right], \quad \delta>0,
$$

$\forall t \in[a, b] \subseteq[0, \infty)$

We need 
Definition 3.9. Here $C_{+}([a, b]):=\left\{f:[a, b] \subseteq[0, \infty) \rightarrow \mathbb{R}_{+}\right.$, continuous functions $\}$. Let $L_{N}: C_{+}([a, b]) \rightarrow C_{+}([a, b])$, operators, $\forall N \in \mathbb{N}$, such that

(i)

$$
L_{N}(\alpha f)=\alpha L_{N}(f), \forall \alpha \geq 0, \forall f \in C_{+}([a, b]),
$$

(ii) if $f, g \in C_{+}([a, b]): f \leq g$, then

$$
L_{N}(f) \leq L_{N}(g), \forall N \in \mathbb{N},
$$

(iii)

$$
L_{N}(f+g) \leq L_{N}(f)+L_{N}(g), \quad \forall f, g \in C_{+}([a, b]) .
$$

We call $\left\{L_{N}\right\}_{N \in \mathbb{N}}$ positive sublinear operators.

We need a Hölder's type inequality, see next:

Theorem 3.10. (see [2]) Let $L: C_{+}([a, b]) \rightarrow C_{+}([a, b])$, be a positive sublinear operator and $f, g \in C_{+}([a, b])$, furthermore let $p, q>1: \frac{1}{p}+\frac{1}{q}=1$. Assume that $L\left((f(\cdot))^{p}\right)\left(s_{*}\right), L\left((g(\cdot))^{q}\right)\left(s_{*}\right)>0$ for some $s_{*} \in[a, b]$. Then

$$
L(f(\cdot) g(\cdot))\left(s_{*}\right) \leq\left(L\left((f(\cdot))^{p}\right)\left(s_{*}\right)\right)^{\frac{1}{p}}\left(L\left((g(\cdot))^{q}\right)\left(s_{*}\right)\right)^{\frac{1}{q}} .
$$

We make

Remark 3.11. By [4], p. 17, we get: let $f, g \in C_{+}([a, b])$, then

$$
\left|L_{N}(f)(x)-L_{N}(g)(x)\right| \leq L_{N}(|f-g|)(x), \quad \forall x \in[a, b] \subseteq[0, \infty) .
$$

Furthermore, we also have that

$$
\left|L_{N}(f)(x)-f(x)\right| \leq L_{N}(|f(\cdot)-f(x)|)(x)+|f(x)|\left|L_{N}\left(e_{0}\right)(x)-1\right|,
$$

$\forall x \in[a, b] \subseteq[0, \infty) ; e_{0}(t)=1$.

From now on we assume that $L_{N}(1)=1$. Hence it holds

$$
\left|L_{N}(f)(x)-f(x)\right| \leq L_{N}(|f(\cdot)-f(x)|)(x), \quad \forall x \in[a, b] \subseteq[0, \infty) .
$$

Next we use Corollary 3.8.

Here $D_{\alpha} f(x)=0$ for a specific $x \in[a, b] \subseteq[0, \infty)$. We also assume that $L_{N}\left(|\cdot-x|^{\alpha+1}\right)(x), L_{N}\left((\cdot-x)^{2(\alpha+1)}\right)(x)>0$. By (3.23) we have

$$
|f(\cdot)-f(x)| \leq \frac{\omega_{1}^{\alpha}\left(D_{\alpha} f, \delta\right)}{\alpha}\left[|\cdot-x|^{\alpha}+\frac{|\cdot-x|^{2 \alpha}}{2 \delta}\right], \quad \delta>0,
$$

true over $[a, b] \subseteq[0, \infty)$.

By (3.30) we get

$$
\begin{gathered}
\left|L_{N}(f)(x)-f(x)\right| \leq \frac{\omega_{1}^{\alpha}\left(D_{\alpha} f, \delta\right)}{\alpha}\left[L_{N}\left(|\cdot-x|^{\alpha}\right)(x)+\frac{L_{N}\left(|\cdot-x|^{2 \alpha}\right)(x)}{2 \delta}\right] \\
\stackrel{(\text { by }(3.27))}{\leq} \frac{\omega_{1}^{\alpha}\left(D_{\alpha} f, \delta\right)}{\alpha}\left[\left(L_{N}\left(|\cdot-x|^{\alpha+1}\right)(x)\right)^{\frac{\alpha}{\alpha+1}}+\frac{\left(L_{N}\left((\cdot-x)^{2(\alpha+1)}\right)(x)\right)^{\frac{\alpha}{\alpha+1}}}{2 \delta}\right]
\end{gathered}
$$


$\left(\right.$ choose $\delta:=\left(\left(L_{N}\left((\cdot-x)^{2(\alpha+1)}\right)(x)\right)^{\frac{\alpha}{\alpha+1}}\right)^{\frac{1}{2}}>0$, hence

$$
\begin{gathered}
\left.\delta^{2}=\left(L_{N}\left((\cdot-x)^{2(\alpha+1)}\right)(x)\right)^{\frac{\alpha}{\alpha+1}}\right) \\
=\frac{\omega_{1}^{\alpha}\left(D_{\alpha} f,\left(L_{N}\left((\cdot-x)^{2(\alpha+1)}\right)(x)\right)^{\frac{\alpha}{2(\alpha+1)}}\right)}{\alpha} . \\
{\left[\left(L_{N}\left(|\cdot-x|^{\alpha+1}\right)(x)\right)^{\frac{\alpha}{\alpha+1}}+\frac{1}{2}\left(L_{N}\left((\cdot-x)^{2(\alpha+1)}\right)(x)\right)^{\frac{\alpha}{2(\alpha+1)}}\right] .}
\end{gathered}
$$

We have proved:

Theorem 3.12. Let $\alpha \in(0,1],[a, b] \subseteq[0, \infty)$. Suppose $f$ is $\alpha$-conformable fractional differentiable on $[a, b] . D_{\alpha} f$ is continuous on $[a, b]$. Let an $x \in[a, b]$ such that $D_{\alpha} f(x)=0$, and $L_{N}: C_{+}([a, b])$ into itself, positive sublinear operators. Assume that $L_{N}(1)=1$ and $L_{N}\left(|\cdot-x|^{\alpha+1}\right)(x), L_{N}\left((\cdot-x)^{2(\alpha+1)}\right)(x)>0, \forall N \in \mathbb{N}$.

Then

$$
\begin{gathered}
\left|L_{N}(f)(x)-f(x)\right| \leq \frac{\omega_{1}^{\alpha}\left(D_{\alpha} f,\left(L_{N}\left((\cdot-x)^{2(\alpha+1)}\right)(x)\right)^{\frac{\alpha}{2(\alpha+1)}}\right)}{\alpha} . \\
{\left[\left(L_{N}\left(|\cdot-x|^{\alpha+1}\right)(x)\right)^{\frac{\alpha}{\alpha+1}}+\frac{1}{2}\left(L_{N}\left((\cdot-x)^{2(\alpha+1)}\right)(x)\right)^{\frac{\alpha}{2(\alpha+1)}}\right], \quad \forall N \in \mathbb{N} .}
\end{gathered}
$$

We make

Remark 3.13. By Theorem 3.10, we get that

$$
L_{N}\left(|\cdot-x|^{\alpha+1}\right)(x) \leq\left(L_{N}\left((\cdot-x)^{2(\alpha+1)}\right)(x)\right)^{\frac{1}{2}} .
$$

As $N \rightarrow+\infty$, by $(3.35)$ and $(3.36)$, and $L_{N}\left((\cdot-x)^{2(\alpha+1)}\right)(x) \rightarrow 0$, we obtain that $L_{N}(f)(x) \rightarrow f(x)$.

We continue with

Remark 3.14. In the assumptions of Corollary 3.7 and (3.22) we can write over $[a, b] \subseteq$ $[0, \infty)$, that

$$
|f(\cdot)-f(x)| \leq \frac{\omega_{1}^{\alpha}\left(D_{\alpha}^{n} f, \delta\right)}{\alpha^{n} n !}\left[|\cdot-x|^{n \alpha}+\frac{|\cdot-x|^{(n+1) \alpha}}{(n+1) \delta}\right], \quad \delta>0 .
$$

By (3.30) we get

$$
\begin{gathered}
\left|L_{N}(f)(x)-f(x)\right| \leq \frac{\omega_{1}^{\alpha}\left(D_{\alpha}^{n} f, \delta\right)}{\alpha^{n} n !} \\
{\left[L_{N}\left(|\cdot-x|^{n \alpha}\right)(x)+\frac{1}{(n+1) \delta} L_{N}\left(|\cdot-x|^{(n+1) \alpha}\right)(x)\right]} \\
\stackrel{\text { by }(3.27))}{\leq} \frac{\omega_{1}^{\alpha}\left(D_{\alpha}^{n} f, \delta\right)}{\alpha^{n} n !}
\end{gathered}
$$




$$
\left[\left(L_{N}\left(|\cdot-x|^{n(\alpha+1)}\right)(x)\right)^{\frac{\alpha}{\alpha+1}}+\frac{1}{(n+1) \delta}\left(L_{N}\left((\cdot-x)^{(n+1)(\alpha+1)}\right)(x)\right)^{\frac{\alpha}{\alpha+1}}\right]
$$

[(here is assumed $L_{N}(1)=1$, and $L_{N}\left(|\cdot-x|^{n(\alpha+1)}\right)(x)$,

$\left.L_{N}\left((\cdot-x)^{(n+1)(\alpha+1)}\right)(x)>0, \forall N \in \mathbb{N}\right)$,

(we take $\delta:=\left(L_{N}\left((\cdot-x)^{(n+1)(\alpha+1)}\right)(x)\right)^{\frac{\alpha}{(n+1)(\alpha+1)}}>0$, then

$\left.\left.\delta^{n+1}=\left(L_{N}\left((\cdot-x)^{(n+1)(\alpha+1)}\right)(x)\right)^{\frac{\alpha}{\alpha+1}}\right)\right]$

$$
=\frac{\omega_{1}^{\alpha}\left(D_{\alpha}^{n} f,\left(L_{N}\left((\cdot-x)^{(n+1)(\alpha+1)}\right)(x)\right)^{\frac{\alpha}{(n+1)(\alpha+1)}}\right)}{\alpha^{n} n !} .
$$

$$
\left[\left(L_{N}\left(|\cdot-x|^{n(\alpha+1)}\right)(x)\right)^{\frac{\alpha}{\alpha+1}}+\frac{1}{(n+1)}\left(L_{N}\left((\cdot-x)^{(n+1)(\alpha+1)}\right)(x)\right)^{\frac{n \alpha}{(n+1)(\alpha+1)}}\right]
$$

We have proved

Theorem 3.15. Let $\alpha \in(0,1], n \in \mathbb{N}$. Suppose $f$ is $n$ times conformable $\alpha$-fractional differentiable on $[a, b] \subseteq[0, \infty)$, and $D_{\alpha}^{n} f$ is continuous on $[a, b]$. For a fixed $x \in[a, b]$ we have $D_{\alpha}^{k} f(x)=0, k=1, \ldots, n$. Let positive sublinear operators $\left\{L_{N}\right\}_{N \in \mathbb{N}}$ from $C_{+}([a, b])$ into itself, such that $L_{N}(1)=1$, and $L_{N}\left(|\cdot-x|^{n(\alpha+1)}\right)(x)$, $L_{N}\left((\cdot-x)^{(n+1)(\alpha+1)}\right)(x)>0, \forall N \in \mathbb{N}$. Then

$$
\begin{aligned}
& \left|L_{N}(f)(x)-f(x)\right| \leq \frac{\omega_{1}^{\alpha}\left(D_{\alpha}^{n} f,\left(L_{N}\left((\cdot-x)^{(n+1)(\alpha+1)}\right)(x)\right)^{\frac{\alpha}{(n+1)(\alpha+1)}}\right)}{\alpha^{n} n !} \cdot \\
& {\left[\left(L_{N}\left(|\cdot-x|^{n(\alpha+1)}\right)(x)\right)^{\frac{\alpha}{\alpha+1}}+\frac{1}{(n+1)}\left(L_{N}\left((\cdot-x)^{(n+1)(\alpha+1)}\right)(x)\right)^{\frac{n \alpha}{(n+1)(\alpha+1)}}\right]}
\end{aligned}
$$

$\forall N \in \mathbb{N}$.

We make

Remark 3.16. By Theorem 3.10, we get that

$$
L_{N}\left(|\cdot-x|^{n(\alpha+1)}\right)(x) \leq\left(L_{N}\left((\cdot-x)^{(n+1)(\alpha+1)}\right)(x)\right)^{\frac{n}{n+1}} .
$$

As $N \rightarrow+\infty$, by $(3.40),(3.41)$, and $L_{N}\left((\cdot-x)^{(n+1)(\alpha+1)}\right)(x) \rightarrow 0$, we derive that $L_{N}(f)(x) \rightarrow f(x)$. 


\section{Applications}

Here we apply Theorems 3.12 and 3.15 to well known Max-product operators.

We make

Remark 4.1. The Max-product Bernstein operators $B_{N}^{(M)}(f)(x)$ are defined by (1.5), see also [4], p. 10; here $f:[0,1] \rightarrow \mathbb{R}_{+}$is a continuous function.

We have $B_{N}^{(M)}(1)=1$, and

$$
B_{N}^{(M)}(|\cdot-x|)(x) \leq \frac{6}{\sqrt{N+1}}, \forall x \in[0,1], \forall N \in \mathbb{N},
$$

see [4], p. 31.

$B_{N}^{(M)}$ are positive sublinear operators and thus they possess the monotonicity property, also since $|\cdot-x| \leq 1$, then $|\cdot-x|^{\beta} \leq 1, \forall x \in[0,1], \forall \beta>0$.

Therefore it holds

$$
B_{N}^{(M)}\left(|\cdot-x|^{1+\beta}\right)(x) \leq \frac{6}{\sqrt{N+1}}, \forall x \in[0,1], \forall N \in \mathbb{N}, \forall \beta>0 .
$$

Furthermore, clearly it holds that

$$
B_{N}^{(M)}\left(|\cdot-x|^{1+\beta}\right)(x)>0, \forall N \in \mathbb{N}, \forall \beta \geq 0 \text { and any } x \in(0,1) .
$$

The operator $B_{N}^{(M)}$ maps $C_{+}([0,1])$ into itself.

We have the following results:

Theorem 4.2. Let $\alpha \in(0,1], f$ is $\alpha$-conformable fractional differentiable on $[0,1]$, $D_{\alpha} f$ is continuous on $[0,1]$. Let $x \in(0,1)$ such that $D_{\alpha} f(x)=0$. Then

$$
\begin{aligned}
& \left|B_{N}^{(M)}(f)(x)-f(x)\right| \leq \frac{\omega_{1}^{\alpha}\left(D_{\alpha} f,\left(\frac{6}{\sqrt{N+1}}\right)^{\frac{\alpha}{2(\alpha+1)}}\right)}{\alpha} . \\
& {\left[\left(\frac{6}{\sqrt{N+1}}\right)^{\frac{\alpha}{\alpha+1}}+\frac{1}{2}\left(\frac{6}{\sqrt{N+1}}\right)^{\frac{\alpha}{2(\alpha+1)}}\right], \quad \forall N \in \mathbb{N} .}
\end{aligned}
$$

Proof. By Theorem 3.12 .

Theorem 4.3. Let $\alpha \in(0,1], f$ is $n$ times conformable $\alpha$-fractional differentiable on $[0,1]$, and $D_{\alpha}^{n} f$ is continuous on $[0,1]$. For a fixed $x \in(0,1)$ we have $D_{\alpha}^{k} f(x)=0$, $k=1, \ldots, n \in \mathbb{N}$. Then

$$
\begin{gathered}
\left|B_{N}^{(M)}(f)(x)-f(x)\right| \leq \frac{\omega_{1}^{\alpha}\left(D_{\alpha}^{n} f,\left(\frac{6}{\sqrt{N+1}}\right)^{\frac{\alpha}{(n+1)(\alpha+1)}}\right)}{\alpha^{n} n !} . \\
{\left[\left(\frac{6}{\sqrt{N+1}}\right)^{\frac{\alpha}{\alpha+1}}+\frac{1}{(n+1)}\left(\frac{6}{\sqrt{N+1}}\right)^{\frac{n \alpha}{(n+1)(\alpha+1)}}\right], \quad \forall N \in \mathbb{N} .}
\end{gathered}
$$

Proof. By Theorem 3.15 . 
Note. By (4.3) and/or (4.4), as $N \rightarrow+\infty$, we get $B_{N}^{(M)}(f)(x) \rightarrow f(x)$.

We continue with

Remark 4.4. The truncated Favard-Szász-Mirakjan operators are given by

$$
T_{N}^{(M)}(f)(x)=\frac{\bigvee_{k=0}^{N} s_{N, k}(x) f\left(\frac{k}{N}\right)}{\bigvee_{k=0}^{N} s_{N, k}(x)}, x \in[0,1], N \in \mathbb{N}, f \in C_{+}([0,1]),
$$

$s_{N, k}(x)=\frac{(N x)^{k}}{k !}$, see also [4], p. 11 .

By [4], p. 178-179, we get that

$$
T_{N}^{(M)}(|\cdot-x|)(x) \leq \frac{3}{\sqrt{N}}, \quad \forall x \in[0,1], \forall N \in \mathbb{N} .
$$

Clearly it holds

$$
T_{N}^{(M)}\left(|\cdot-x|^{1+\beta}\right)(x) \leq \frac{3}{\sqrt{N}}, \forall x \in[0,1], \forall N \in \mathbb{N}, \forall \beta>0 .
$$

The operators $T_{N}^{(M)}$ are positive sublinear operators mapping $C_{+}([0,1])$ into itself, with $T_{N}^{(M)}(1)=1$.

Furthermore it holds

$$
T_{N}^{(M)}\left(|\cdot-x|^{\lambda}\right)(x)=\frac{\bigvee_{k=0}^{N} \frac{(N x)^{k}}{k !}\left|\frac{k}{N}-x\right|^{\lambda}}{\bigvee_{k=0}^{N} \frac{(N x)^{k}}{k !}}>0, \quad \forall x \in(0,1], \forall \lambda \geq 1, \forall N \in \mathbb{N}
$$

We give the following results:

Theorem 4.5. Let $\alpha \in(0,1], f$ is $\alpha$-conformable fractional differentiable on $[0,1]$. $D_{\alpha} f$ is continuous on $[0,1]$. Let $x \in(0,1]$ such that $D_{\alpha} f(x)=0$. Then

$$
\begin{aligned}
& \left|T_{N}^{(M)}(f)(x)-f(x)\right| \leq \frac{\omega_{1}^{\alpha}\left(D_{\alpha} f,\left(\frac{3}{\sqrt{N}}\right)^{\frac{\alpha}{2(\alpha+1)}}\right)}{\alpha} . \\
& \quad\left[\left(\frac{3}{\sqrt{N}}\right)^{\frac{\alpha}{\alpha+1}}+\frac{1}{2}\left(\frac{3}{\sqrt{N}}\right)^{\frac{\alpha}{2(\alpha+1)}}\right], \quad \forall N \in \mathbb{N} .
\end{aligned}
$$

Proof. By Theorem 3.12 .

Theorem 4.6. Let $\alpha \in(0,1], f$ is $n$ times conformable $\alpha$-fractional differentiable on $[0,1]$, and $D_{\alpha}^{n} f$ is continuous on $[0,1]$. For a fixed $x \in(0,1]$ we have $D_{\alpha}^{k} f(x)=0$, $k=1, \ldots, n \in \mathbb{N}$. Then

$$
\begin{aligned}
& \left|T_{N}^{(M)}(f)(x)-f(x)\right| \leq \frac{\omega_{1}^{\alpha}\left(D_{\alpha}^{n} f,\left(\frac{3}{\sqrt{N}}\right)^{\frac{\alpha}{(n+1)(\alpha+1)}}\right)}{\alpha^{n} n !} . \\
& {\left[\left(\frac{3}{\sqrt{N}}\right)^{\frac{\alpha}{\alpha+1}}+\frac{1}{(n+1)}\left(\frac{3}{\sqrt{N}}\right)^{\frac{n \alpha}{(n+1)(\alpha+1)}}\right], \quad \forall N \in \mathbb{N} .}
\end{aligned}
$$

Proof. By Theorem 3.15. 
Note. By (4.9) and/or (4.10), as $N \rightarrow+\infty$, we get $T_{N}^{(M)}(f)(x) \rightarrow f(x)$.

We continue with

Remark 4.7. Next we study the truncated Max-product Baskakov operators (see [4], p. 11)

$$
U_{N}^{(M)}(f)(x)=\frac{\bigvee_{k=0}^{N} b_{N, k}(x) f\left(\frac{k}{N}\right)}{\bigvee_{k=0}^{N} b_{N, k}(x)}, x \in[0,1], f \in C_{+}([0,1]), N \in \mathbb{N},
$$

where

$$
b_{N, k}(x)=\left(\begin{array}{c}
N+k-1 \\
k
\end{array}\right) \frac{x^{k}}{(1+x)^{N+k}} .
$$

From [4], pp. 217-218, we get $(x \in[0,1])$

$$
\left(U_{N}^{(M)}(|\cdot-x|)\right)(x) \leq \frac{2 \sqrt{3}(\sqrt{2}+2)}{\sqrt{N+1}}, N \geq 2, N \in \mathbb{N} .
$$

Let $\lambda \geq 1$, clearly then it holds

$$
\left(U_{N}^{(M)}\left(|\cdot-x|^{\lambda}\right)\right)(x) \leq \frac{2 \sqrt{3}(\sqrt{2}+2)}{\sqrt{N+1}}, \quad \forall N \geq 2, N \in \mathbb{N} .
$$

Also it holds $U_{N}^{(M)}(1)=1$, and $U_{N}^{(M)}$ are positive sublinear operators from $C_{+}([0,1])$ into itself. Furthermore it holds

$$
U_{N}^{(M)}\left(|\cdot-x|^{\lambda}\right)(x)>0, \quad \forall x \in(0,1], \forall \lambda \geq 1, \forall N \in \mathbb{N} .
$$

We give

Theorem 4.8. Let $\alpha \in(0,1], f$ is $\alpha$-conformable fractional differentiable on $[0,1]$. $D_{\alpha} f$ is continuous on $[0,1]$. Let $x \in(0,1]$ such that $D_{\alpha} f(x)=0$. Then

$$
\begin{gathered}
\left|U_{N}^{(M)}(f)(x)-f(x)\right| \leq \frac{\omega_{1}^{\alpha}\left(D_{\alpha} f,\left(\frac{2 \sqrt{3}(\sqrt{2}+2)}{\sqrt{N+1}}\right)^{\frac{\alpha}{2(\alpha+1)}}\right)}{\alpha} . \\
{\left[\left(\frac{2 \sqrt{3}(\sqrt{2}+2)}{\sqrt{N+1}}\right)^{\frac{\alpha}{\alpha+1}}+\frac{1}{2}\left(\frac{2 \sqrt{3}(\sqrt{2}+2)}{\sqrt{N+1}}\right)^{\left.\frac{\alpha}{2(\alpha+1)}\right], \quad \forall N \geq 2, N \in \mathbb{N} .} .\right.}
\end{gathered}
$$

Proof. By Theorem 3.12.

Theorem 4.9. Let $\alpha \in(0,1], f$ is $n$ times conformable $\alpha$-fractional differentiable on $[0,1]$, and $D_{\alpha}^{n} f$ is continuous on $[0,1]$. For a fixed $x \in(0,1]$ we have $D_{\alpha}^{k} f(x)=0$, $k=1, \ldots, n \in \mathbb{N}$. Then

$$
\begin{gathered}
\left|U_{N}^{(M)}(f)(x)-f(x)\right| \leq \frac{\omega_{1}^{\alpha}\left(D_{\alpha}^{n} f,\left(\frac{2 \sqrt{3}(\sqrt{2}+2)}{\sqrt{N+1}}\right)^{\frac{\alpha}{(n+1)(\alpha+1)}}\right)}{\alpha^{n} n !} . \\
{\left[\left(\frac{2 \sqrt{3}(\sqrt{2}+2)}{\sqrt{N+1}}\right)^{\frac{\alpha}{\alpha+1}}+\frac{1}{(n+1)}\left(\frac{2 \sqrt{3}(\sqrt{2}+2)}{\sqrt{N+1}}\right)^{\frac{n \alpha}{(n+1)(\alpha+1)}}\right],}
\end{gathered}
$$


$\forall N \geq 2, N \in \mathbb{N}$.

Proof. By Theorem 3.15 .

Note. By (4.16) and/or (4.17), as $N \rightarrow+\infty$, we get that $U_{N}^{(M)}(f)(x) \rightarrow f(x)$.

We continue with

Remark 4.10. Here we study the Max-product Meyer-Köning and Zeller operators (see [4], p. 11) defined by

$$
\begin{aligned}
& Z_{N}^{(M)}(f)(x)=\frac{\bigvee_{k=0}^{\infty} s_{N, k}(x) f\left(\frac{k}{N+k}\right)}{\bigvee_{k=0}^{\infty} s_{N, k}(x)}, \forall N \in \mathbb{N}, f \in C_{+}([0,1]), \\
& s_{N, k}(x)=\left(\begin{array}{c}
N+k \\
k
\end{array}\right) x^{k}, x \in[0,1] .
\end{aligned}
$$

By [4], p. 253, we get that

$$
Z_{N}^{(M)}(|\cdot-x|)(x) \leq \frac{8(1+\sqrt{5})}{3} \frac{\sqrt{x}(1-x)}{\sqrt{N}}, \forall x \in[0,1], \forall N \geq 4, N \in \mathbb{N} .
$$

As before we get that (for $\lambda \geq 1$ )

$$
Z_{N}^{(M)}\left(|\cdot-x|^{\lambda}\right)(x) \leq \frac{8(1+\sqrt{5})}{3} \frac{\sqrt{x}(1-x)}{\sqrt{N}}:=\rho(x),
$$

$\forall x \in[0,1], N \geq 4, N \in \mathbb{N}$.

Also it holds $Z_{N}^{(M)}(1)=1$, and $Z_{N}^{(M)}$ are positive sublinear operators from $C_{+}([0,1])$ into itself. Also it holds

$$
Z_{N}^{(M)}\left(|\cdot-x|^{\lambda}\right)(x)>0, \quad \forall x \in(0,1), \forall \lambda \geq 1, \forall N \in \mathbb{N} .
$$

We give

Theorem 4.11. Let $\alpha \in(0,1], f$ is $\alpha$-conformable fractional differentiable on $[0,1]$. $D_{\alpha} f$ is continuous on $[0,1]$. Let $x \in(0,1)$ such that $D_{\alpha} f(x)=0$. Then

$$
\begin{aligned}
& \left|Z_{N}^{(M)}(f)(x)-f(x)\right| \leq \frac{\omega_{1}^{\alpha}\left(D_{\alpha} f,(\rho(x))^{\frac{\alpha}{2(\alpha+1)}}\right)}{\alpha} . \\
& {\left[(\rho(x))^{\frac{\alpha}{\alpha+1}}+\frac{1}{2}(\rho(x))^{\frac{\alpha}{2(\alpha+1)}}\right], \quad \forall N \geq 4, N \in \mathbb{N} .}
\end{aligned}
$$

Proof. By Theorem 3.12 .

Theorem 4.12. Let $\alpha \in(0,1], f$ is $n$ times conformable $\alpha$-fractional differentiable on $[0,1]$, and $D_{\alpha}^{n} f$ is continuous on $[0,1]$. For a fixed $x \in(0,1)$ we have $D_{\alpha}^{k} f(x)=0$, $k=1, \ldots, n \in \mathbb{N}$. Then

$$
\begin{gathered}
\left|Z_{N}^{(M)}(f)(x)-f(x)\right| \leq \frac{\omega_{1}^{\alpha}\left(D_{\alpha}^{n} f,(\rho(x))^{\frac{\alpha}{(n+1)(\alpha+1)}}\right)}{\alpha^{n} n !} . \\
{\left[(\rho(x))^{\frac{\alpha}{\alpha+1}}+\frac{1}{(n+1)}(\rho(x))^{\frac{n \alpha}{(n+1)(\alpha+1)}}\right], \quad \forall N \geq 4, N \in \mathbb{N} .}
\end{gathered}
$$


Proof. By Theorem 3.15.

Note. By (4.22) and/or (4.23), as $N \rightarrow+\infty$, we get that $Z_{N}^{(M)}(f)(x) \rightarrow f(x)$.

We continue with

Remark 4.13. Here we deal with the Max-product truncated sampling operators (see [4], p. 13) defined by

$$
W_{N}^{(M)}(f)(x)=\frac{\bigvee_{k=0}^{N} \frac{\sin (N x-k \pi)}{N x-k \pi} f\left(\frac{k \pi}{N}\right)}{\bigvee_{k=0}^{N} \frac{\sin (N x-k \pi)}{N x-k \pi}},
$$

and

$$
K_{N}^{(M)}(f)(x)=\frac{\bigvee_{k=0}^{N} \frac{\sin ^{2}(N x-k \pi)}{(N x-k \pi)^{2}} f\left(\frac{k \pi}{N}\right)}{\bigvee_{k=0}^{N} \frac{\sin ^{2}(N x-k \pi)}{(N x-k \pi)^{2}}},
$$

$\forall x \in[0, \pi], f:[0, \pi] \rightarrow \mathbb{R}_{+}$a continuous function.

Following [4], p. 343, and making the convention $\frac{\sin (0)}{0}=1$ and denoting $s_{N, k}(x)=\frac{\sin (N x-k \pi)}{N x-k \pi}$, we get that $s_{N, k}\left(\frac{k \pi}{N}\right)=1$, and $s_{N, k}\left(\frac{j \pi}{N}\right)=0$, if $k \neq j$, furthermore $W_{N}^{(M)}(f)\left(\frac{j \pi}{N}\right)=f\left(\frac{j \pi}{N}\right)$, for all $j \in\{0, \ldots, N\}$.

Clearly $W_{N}^{(M)}(f)$ is a well-defined function for all $x \in[0, \pi]$, and it is continuous on $[0, \pi]$, also $W_{N}^{(M)}(1)=1$.

By [4], p. 344, $W_{N}^{(M)}$ are positive sublinear operators.

Call $I_{N}^{+}(x)=\left\{k \in\{0,1, \ldots, N\} ; s_{N, k}(x)>0\right\}$, and set $x_{N, k}:=\frac{k \pi}{N}, k \in$ $\{0,1, \ldots, N\}$.

We see that

$$
W_{N}^{(M)}(f)(x)=\frac{\bigvee_{k \in I_{N}^{+}(x)} s_{N, k}(x) f\left(x_{N, k}\right)}{\bigvee_{k \in I_{N}^{+}(x)} s_{N, k}(x)} .
$$

By [4], p. 346, we have

$$
W_{N}^{(M)}(|\cdot-x|)(x) \leq \frac{\pi}{2 N}, \quad \forall N \in \mathbb{N}, \forall x \in[0, \pi] .
$$

Notice also $\left|x_{N, k}-x\right| \leq \pi, \forall x \in[0, \pi]$.

Therefore $(\lambda \geq 1)$ it holds

$$
W_{N}^{(M)}\left(|\cdot-x|^{\lambda}\right)(x) \leq \frac{\pi^{\lambda-1} \pi}{2 N}=\frac{\pi^{\lambda}}{2 N}, \quad \forall x \in[0, \pi], \forall N \in \mathbb{N} .
$$

If $x \in\left(\frac{j \pi}{N}, \frac{(j+1) \pi}{N}\right)$, with $j \in\{0,1, \ldots, N\}$, we obtain $n x-j \pi \in(0, \pi)$ and thus

$$
s_{N, j}(x)=\frac{\sin (N x-j \pi)}{N x-j \pi}>0,
$$

see [4], pp. 343-344.

Consequently it holds $(\lambda \geq 1)$

$$
W_{N}^{(M)}\left(|\cdot-x|^{\lambda}\right)(x)=\frac{\bigvee_{k \in I_{N}^{+}(x)} s_{N, k}(x)\left|x_{N, k}-x\right|^{\lambda}}{\bigvee_{k \in I_{N}^{+}(x)} s_{N, k}(x)}>0, \quad \forall x \in[0, \pi],
$$


such that $x \neq x_{N, k}$, for any $k \in\{0,1, \ldots, N\}$.

We give

Theorem 4.14. Let $\alpha \in(0,1], f$ is $\alpha$-conformable fractional differentiable on $[0, \pi]$. $D_{\alpha} f$ is continuous on $[0, \pi]$. Let $x \in[0, \pi]$ be such that $x \neq \frac{k \pi}{N}, k \in\{0,1, \ldots, N\}, \forall$ $N \in \mathbb{N}$, and $D_{\alpha} f(x)=0$. Then

$$
\begin{gathered}
\left|W_{N}^{(M)}(f)(x)-f(x)\right| \leq \frac{\omega_{1}^{\alpha}\left(D_{\alpha} f,\left(\frac{\pi^{2(\alpha+1)}}{2 N}\right)^{\frac{\alpha}{2(\alpha+1)}}\right)}{\alpha} . \\
{\left[\left(\frac{\pi^{\alpha+1}}{2 N}\right)^{\frac{\alpha}{\alpha+1}}+\frac{1}{2}\left(\frac{\pi^{2(\alpha+1)}}{2 N}\right)^{\frac{\alpha}{2(\alpha+1)}}\right]=} \\
\frac{\omega_{1}^{\alpha}\left(D_{\alpha} f, \frac{\pi^{\alpha}}{(2 N)^{\frac{\alpha}{2(\alpha+1)}}}\right)}{\alpha}\left[\frac{\pi^{\alpha}}{(2 N)^{\frac{\alpha}{(\alpha+1)}}}+\frac{\pi^{\alpha}}{2(2 N)^{\frac{\alpha}{2(\alpha+1)}}}\right], \quad \forall N \in \mathbb{N} .
\end{gathered}
$$

Proof. By Theorem 3.12.

Theorem 4.15. Let $\alpha \in(0,1], n \in \mathbb{N}$. Suppose $f$ is $n$ times conformable $\alpha$-fractional differentiable on $[0, \pi]$, and $D_{\alpha}^{n} f$ is continuous on $[0, \pi]$. For a fixed $x \in[0, \pi]: x \neq \frac{k \pi}{N}$, $k \in\{0,1, \ldots, N\}, \forall N \in \mathbb{N}$, we have $D_{\alpha}^{k} f(x)=0, k=1, \ldots, n$. Then

$$
\begin{aligned}
& \left|W_{N}^{(M)}(f)(x)-f(x)\right| \leq \frac{\omega_{1}^{\alpha}\left(D_{\alpha}^{n} f, \frac{\pi^{\alpha}}{(2 N)^{(n+1)(\alpha+1)}}\right)}{\alpha^{n} n !} . \\
& {\left[\frac{\pi^{n \alpha}}{(2 N)^{\frac{\alpha}{(\alpha+1)}}}+\frac{\pi^{n \alpha}}{(n+1)(2 N)^{\frac{n \alpha}{(n+1)(\alpha+1)}}}\right], \quad \forall N \in \mathbb{N} .}
\end{aligned}
$$

Proof. By Theorem 3.15.

Note. (i) if $x=\frac{j \pi}{N}, j \in\{0, \ldots, N\}$, then the left hand sides of (4.30) and (4.31) are zero, so these inequalities are trivially valid.

(ii) from (4.30) and/or (4.31), as $N \rightarrow+\infty$, we get that $W_{N}^{(M)}(f)(x) \rightarrow f(x)$.

We make

Remark 4.16. Here we continue with the Max-product truncated sampling operators (see [4], p. 13) defined by

$$
K_{N}^{(M)}(f)(x)=\frac{\bigvee_{k=0}^{N} \frac{\sin ^{2}(N x-k \pi)}{(N x-k \pi)^{2}} f\left(\frac{k \pi}{N}\right)}{\bigvee_{k=0}^{N} \frac{\sin ^{2}(N x-k \pi)}{(N x-k \pi)^{2}}},
$$

$\forall x \in[0, \pi], f:[0, \pi] \rightarrow \mathbb{R}_{+}$a continuous function.

Following [4], p. 350, and making the convention $\frac{\sin (0)}{0}=1$ and denoting

$$
s_{N, k}(x)=\frac{\sin ^{2}(N x-k \pi)}{(N x-k \pi)^{2}},
$$


we get that $s_{N, k}\left(\frac{k \pi}{N}\right)=1$, and $s_{N, k}\left(\frac{j \pi}{N}\right)=0$, if $k \neq j$, furthermore

$$
K_{N}^{(M)}(f)\left(\frac{j \pi}{N}\right)=f\left(\frac{j \pi}{N}\right)
$$

for all $j \in\{0, \ldots, N\}$.

Since $s_{N, j}\left(\frac{j \pi}{N}\right)=1$ it follows that

$$
\bigvee_{k=0}^{N} s_{N, k}\left(\frac{j \pi}{N}\right) \geq 1>0
$$

for all $j \in\{0,1, \ldots, N\}$. Hence $K_{N}^{(M)}(f)$ is well-defined function for all $x \in[0, \pi]$, and it is continuous on $[0, \pi]$, also $K_{N}^{(M)}(1)=1$. By [4], p. $350, K_{N}^{(M)}$ are positive sublinear operators.

Denote $x_{N, k}:=\frac{k \pi}{N}, k \in\{0,1, \ldots, N\}$.

By [4], p. 352, we have

$$
K_{N}^{(M)}(|\cdot-x|)(x) \leq \frac{\pi}{2 N}, \quad \forall N \in \mathbb{N}, \forall x \in[0, \pi] .
$$

Notice also $\left|x_{N, k}-x\right| \leq \pi, \forall x \in[0, \pi]$.

Therefore $(\lambda \geq 1)$ it holds

$$
K_{N}^{(M)}\left(|\cdot-x|^{\lambda}\right)(x) \leq \frac{\pi^{\lambda-1} \pi}{2 N}=\frac{\pi^{\lambda}}{2 N}, \quad \forall x \in[0, \pi], \forall N \in \mathbb{N} .
$$

If $x \in\left(\frac{j \pi}{N}, \frac{(j+1) \pi}{N}\right)$, with $j \in\{0,1, \ldots, N\}$, we obtain $n x-j \pi \in(0, \pi)$ and thus

$$
s_{N, j}(x)=\frac{\sin ^{2}(N x-j \pi)}{(N x-j \pi)^{2}}>0,
$$

see [4], pp. 350 .

Consequently it holds $(\lambda \geq 1)$

$$
K_{N}^{(M)}\left(|\cdot-x|^{\lambda}\right)(x)=\frac{\bigvee_{k=0}^{N} s_{N, k}(x)\left|x_{N, k}-x\right|^{\lambda}}{\bigvee_{k=0}^{N} s_{N, k}(x)}>0, \quad \forall x \in[0, \pi],
$$

such that $x \neq x_{N, k}$, for any $k \in\{0,1, \ldots, N\}$.

We give

Theorem 4.17. Let $\alpha \in(0,1], f$ is $\alpha$-conformable fractional differentiable on $[0, \pi]$. $D_{\alpha} f$ is continuous on $[0, \pi]$. Let $x \in[0, \pi]$ be such that $x \neq \frac{k \pi}{N}, k \in\{0,1, \ldots, N\}, \forall$ $N \in \mathbb{N}$, and $D_{\alpha} f(x)=0$. Then

$$
\begin{gathered}
\left|K_{N}^{(M)}(f)(x)-f(x)\right| \leq \frac{\omega_{1}^{\alpha}\left(D_{\alpha} f,\left(\frac{\pi^{2(\alpha+1)}}{2 N}\right)^{\frac{\alpha}{2(\alpha+1)}}\right)}{\alpha} \\
\cdot\left[\left(\frac{\pi^{\alpha+1}}{2 N}\right)^{\frac{\alpha}{\alpha+1}}+\frac{1}{2}\left(\frac{\pi^{2(\alpha+1)}}{2 N}\right)^{\frac{\alpha}{2(\alpha+1)}}\right]
\end{gathered}
$$




$$
=\frac{\omega_{1}^{\alpha}\left(D_{\alpha} f, \frac{\pi^{\alpha}}{(2 N)^{\frac{\alpha}{2(\alpha+1)}}}\right)}{\alpha}\left[\frac{\pi^{\alpha}}{(2 N)^{\frac{\alpha}{(\alpha+1)}}}+\frac{\pi^{\alpha}}{2(2 N)^{\frac{\alpha}{2(\alpha+1)}}}\right], \quad \forall N \in \mathbb{N} .
$$

Proof. By Theorem 3.12 .

Theorem 4.18. Let $\alpha \in(0,1], n \in \mathbb{N}$. Suppose $f$ is $n$ times conformable $\alpha$-fractional differentiable on $[0, \pi]$, and $D_{\alpha}^{n} f$ is continuous on $[0, \pi]$. For a fixed $x \in[0, \pi]: x \neq \frac{k \pi}{N}$, $k \in\{0,1, \ldots, N\}, \forall N \in \mathbb{N}$, we have $D_{\alpha}^{k} f(x)=0, k=1, \ldots, n$. Then

$$
\begin{aligned}
& \left|K_{N}^{(M)}(f)(x)-f(x)\right| \leq \frac{\omega_{1}^{\alpha}\left(D_{\alpha}^{n} f, \frac{\pi^{\alpha}}{(2 N)^{\frac{\alpha}{(n+1)(\alpha+1)}}}\right)}{\alpha^{n} n !} \\
& \cdot\left[\frac{\pi^{n \alpha}}{(2 N)^{\frac{\alpha}{(\alpha+1)}}}+\frac{\pi^{n \alpha}}{(n+1)(2 N)^{\frac{n \alpha}{(n+1)(\alpha+1)}}}\right], \quad \forall N \in \mathbb{N} .
\end{aligned}
$$

Proof. By Theorem 3.15.

Note. (i) if $x=\frac{j \pi}{N}, j \in\{0, \ldots, N\}$, then the left hand sides of (4.36) and (4.37) are zero, so these inequalities are trivially valid.

(ii) from (4.36) and/or (4.37), as $N \rightarrow+\infty$, we get that $K_{N}^{(M)}(f)(x) \rightarrow f(x)$.

\section{References}

[1] Abu Hammad, M., Khalil, R., Abel's formula and Wronskian for conformable fractional differential equations, International J. Differential Equations Appl., 13, 3(2014), 177-183.

[2] Anastassiou, G., Approximation by Sublinear Operators, submitted, 2017.

[3] Anderson, D., Taylor's formula and integral inequalities for conformable fractional derivatives, Contributions in Mathematics and Engineering, in Honor of Constantin Carathéodory, Springer, Berlin, (2016), pp. 25-43.

[4] Bede, B., Coroianu, L., Gal, S., Approximation by Max-Product type Operators, Springer, Heidelberg, New York, 2016.

[5] Iyiola, O., Nwaeze, E., Some New Results on the new Conformable Fractional Calculus with Application using D'Alambert approach, Progr. Fract. Differ. Appl., 2, 2(2016), 115122 .

[6] Khalil, R., Al Horani, M., Yousef, A., Sababheh, M., A new definition of fractional derivative, J. Computational Appl. Math., 264(2014), 65-70.

[7] Lorentz, G.G., Bernstein Polynomials, Chelsea Publishing Company, New ork, NY, 1986, 2nd edition.

[8] Popoviciu, T., Sur l'approximation de fonctions convexes d'order superieur, Mathematica (Cluj), 10(1935), 49-54.

George A. Anastassiou

Department of Mathematical Sciences

University of Memphis

Memphis, TN 38152, U.S.A.

e-mail: ganastss@memphis.edu 